\section{PTH-126 NEOPLASIA PROGRESSION IN BARRETT'S OESOPHAGUS WITH BASELINE LOW-GRADE DYSPLASIA UNDERGOING RADIOFREQUENCY ABLATION}

1,2 Jose Santiago-García*, 1,2Jacobo Ortiz Fernández-Sordo, 'Philip Kaye, 1,2Mirela Pana, 1,2Krish Ragunath. 'Nottingham University Hospitals NHS Trust, Nottingham, UK; ${ }^{2}$ Nottingham Digestive Diseases Centre, NIHR Biomedical Research Centre, University of Nottingham, Nottingham, UK

\subsection{6/gutjnl-2018-BSGAbstracts.300}

Introduction Barrett's oesophagus (BO) with low-grade dysplasia (LGD) can progress to high-grade dysplasia (HGD) and oesophageal adenocarcinoma (OAC). For this reason confirmed LGD has been recently approved by NICE as an indication for radiofrequency ablation (RFA). We aimed to evaluate the progression rate among LGD patients undergoing RFA and thus corroborate the importance of the ablative treatment in this scenario.

Methods Review of the RFA database in a tertiary referral centre over the period 2008-2018 was done. Demographics, BO characteristics and RFA treatment features were collected and included for analysis. Patients with an indication for RFA other than BO with LGD were excluded. Only endoscopies done during the RFA or exit-biopsies period were taken into consideration.

Results 41 patients were included for analysis: 31 males (75.6\%), mean age $66(\mathrm{SD}=10)$, median $\mathrm{BO}$ length 6.6 $(\mathrm{SD}=3.2)$. In 34 patients $(82.9 \%)$ LGD was detected through random biopsies and was confirmed by 2 expert pathologists within an interval of 6 months. 7 patients (17.1\%) presenting a visible lesion were diagnosed following endoscopic mucosal resection (EMR).

11 patients $(26.8 \%)$ completed the RFA treatment with a median number of $2.5(\mathrm{SD}=1)$ sessions achieving complete eradication of dysplasia and intestinal metaplasia. 1 patient $(2.3 \%)$ abandoned the treatment after developing severe comorbidities. 12 patients $(29.2 \%)$ who finished the RFA were awaiting exit-biopsies and $17(41.4 \%)$ still continuing ablative treatment.

LGD progression was detected in 4 patients (9.75\%) with 3 confirmed cases of HGD and one OAC. The median time for progression was $16.7(\mathrm{SD}=4.5)$ months since the confirmed diagnosis. The most common location was the gastro-oesophageal junction, GOJ (75\%). 3 of these cases, including the OAC, presented with a visible lesion and were treated endoscopically (2 EMR; 1 endoscopic submucosal dissection, ESD). 2 additional RFA sessions were applied to the GOJ progression presented as non-visible lesion.

Conclusions The progression rate among BO patients with confirmed LGD undergoing RFA in our series was $9.75 \%$. This data emphasises the high risk of progression presented by this subgroup and supports its indication for ablative treatment.

$\begin{aligned} & \text { Abstract PTH-126 Table } 1 \\
& \text { with progression }\end{aligned}$
\begin{tabular}{|cccccc} 
Case & Age & Sex & $\begin{array}{c}\text { Bosline characteristics of the patients } \\
\text { length } \\
\text { (cm) }\end{array}$ & $\begin{array}{c}\text { Visible } \\
\text { lesion } \\
\text { pre-RFA }\end{array}$ & $\begin{array}{c}\text { Number } \\
\text { of RFA } \\
\text { sessions }\end{array}$ \\
1 & 71 & F & 4 & Yes & 2 \\
$\mathbf{2}$ & 47 & M & 11 & Yes & 2 \\
3 & 80 & M & 2 & No & 2 \\
4 & 79 & M & 2 & No & 2
\end{tabular}

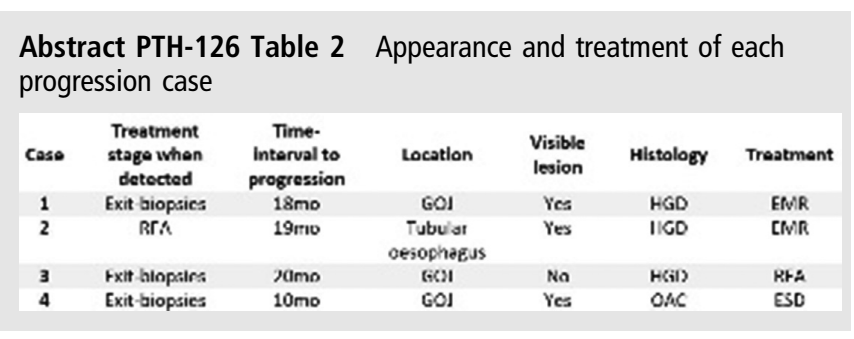

\section{PTH-127 STUDIES OF SALIVARY PEPSIN IN PATIENTS WITH REFLUX DISEASE}

Caroline Race*. Sheffield Teaching Hositals NHS FT, Sheffield, UK

\subsection{6/gutjnl-2018-BSGAbstracts.301}

Introduction Salivary pepsin has been proposed as a non-invasive diagnostic marker of reflux disease but results have been conflicting. Our aim was therefore to study the optimal timing of salivary sampling.

Methods Asymptomatic volunteers and patients with reflux symptoms being considered for antireflux surgery were studied by structured questionnaire, oesophageal $\mathrm{pH}$ (modified to alarm with proximal reflux events), HRM, oesophageal impedance and multiple timed salivary samples after reflux symptoms, when the proximal reflux alarm was activated, on rising and after the evening meal. After an initial pilot, utilising only Peptest analysis, samples were coded and split for analysis by Peptest and in-house ELISA.

Results Twenty volunteers (6 males, aged 21-56 years) and thirty patients (18 males, aged $20-72$ years) were studied. Volunteers had normal acid exposure $(1.0,0-3.5 \%)$ and impedance values. Patients had increased acid exposure $(7.3,0.2-$ $39 \%$ ) and 23 had values in excess of the normal range and were classified as true reflux. A further three had significant non-acid reflux and the final four were excluded from further analysis. In patients with true acid reflux, 20 reported symptoms during the 24 hour period and in 12 the proximal reflux alarm was triggered.

Peptest analysis of 276 samples from the 20 volunteers revealed detectable levels in 102 samples (37\%). 75\% of volunteers had at least one positive sample (range 1-18). Peptest analysis of 458 samples from patients with true reflux revealed positive results in $41 \%$ of samples. $84 \%$ of patients had at least one positive result. The indirect ELISA of samples from volunteers revealed detectable levels in 87\%. All volunteers had at least one positive sample. ELISA of samples from patients with true reflux revealed positive results 79\%. All patients had at least one positive result.

Peptest gave a significant difference in concentration between the control and reflux groups, but with controls giving the higher readings ( $\mathrm{t}$-test, $\mathrm{p}<0.01$ ). In contrast, there was no significant difference in the average ELISA score for pepsin concentration in each group. Peptest quantitations were not significantly different between patients and volunteers on rising but were significantly lower in patients post-prandially $(\mathrm{p}<0.05)$. Peptest results showed no difference in salivary pepsin following the reflux alarm and symptomatic individuals having lower values than volunteers.

Conclusions Salivary pepsin does not discriminate between volunteers and patients with reflux disease. Furthermore, we demonstrate inconsistencies between an in house validated ELISA for salivary pepsin and commercially available Peptest. 